\title{
The study on Translation Quality Assessment in the Translation Contests Based on Malcolm Williams' Argumentation-centered Translation Quality Assessment Model
}

\author{
Xiyun Liu', a , Youbin Zhao ${ }^{2, b}$ \\ ${ }^{1}$ College of Foreign Studies, Jinan University, Guangdong 510632, China; \\ ${ }^{2}$ School of Translation Studies, Jinan University, Guangdong 519070, China. \\ a liu.xiyun@foxmail.com, ${ }^{b}$ youbinz@jnu.edu.cn
}

Keywords: Translation quality assessment, Translation contest, Malcolm Williams' argumentation-centered translation quality assessment Model.

\begin{abstract}
The translation contest is the main form of evaluating the translators' ability and selecting the gifted translators. In the translation contest, a systematic and overall translation quality assessment is always needed. With the purpose of providing the comparatively scientific translation quality assessment for the translation contests, this paper develops a quantitative evaluation approach by applying Malcolm Williams' argumentation-centered translation quality assessment model, which can make the final evaluation more convincible and enhance the authority and the fairness of the contest.
\end{abstract}

\section{Introduction}

In recent years, numerous translation contests have been springing up in China since the first translation contest of China, Han Suyin award for Young Translators, was held in 1986, which is also the largest and most influential contest in China. These contests are held by a wide variety of sponsors such as the industry association, the language service enterprises and the universities. And the content of these contests is also involved in many areas, from the literature to the law, from the business to the news media. According to incomplete statistics, there are more than twenty thousand people attending various translation contests in total every year. The translation contests provide an excellent platform for talented translators to demonstrate their splendid ability and practice their translation techniques.

However, such intense passion of the contestants presents a radical challenge to the judgment. It is indeed a tough task because the judges need to select the most outstanding work from so many similar translations as vast as the ocean in the limited time. To solve the challenge mentioned above, generally, judges always draw up a set of principles about the style, the structure, the grammar and then give a point to every sentence according to the established principles. In the end, the summation of every sentence's point can be regarded as the translation's final assessment. This kind of translation quality assessment has obvious defects. On one hand, the judgment process is full of the subjectivity and the principle has the strong arbitrariness. On the other hand, by calculating the errors of every sentence, the method can be only regard as a micro-analysis and ignores the function of the whole discourse. Therefore, it is necessary to explore a more scientific and systematic quantitative approach of the translation quality assessment (TQA) in translation contests.

The paper is advanced under this situation. Section 1 gives a brief introduction of Malcolm Williams' argumentation-centered translation quality assessment model (ARTRAQ) and mainly discusses its core concept--the argument schema. Section 2 explains other argumentation parameters and its unique weighted ARTRAQ grid method. Section 3 applies ARTRAQ to examine a scientific and systematic quantitative approach, combined with the practical requirement of the translation contest. 


\section{Malcolm Williams' argumentation-centered translation quality assessment model and argument schema}

Noticing the imperfections of modern TQA approaches, Malcolm Williams, an associate professor of University of Ottawa, proposed his argumentation-centered translation quality assessment model in 2004. In this model, he applies an approach of discourse analysis - argumentation theory - to TQA and create an assessment framework to replenish existing microtextual schemes. This model can contribute to reduce some of the subjectivity and randomness in the process of evaluating the translation quality.

In his view, argumentation, as a component of rhetoric, is an important manner of writing in a broad range of fields, including both social science and natural science. As a method of discourse analysis, it can reveal the logical connection of every part in a passage and furthermore it can be regarded as a main presentation form of knowledge and meaning. That is to say, once there is content to convey, an argument is present. Therefore, if we want to understand the meaning of a passage, we can analyze the structure of the argument. The process above is called describing the argument schema, the core concept of the theory, which provides a new perspective.

Based on numerous researches, another famous scholar, Toulmin and his colleagues have concluded that the components of an argument are essentially the same in all fields and types of text. Citing their theory, Malcolm Williams agrees that the argument schema of a passage consists of six parts - claims, grounds, warrants, backings, qualifiers and rebuttals (short for B, W, G, C, Q, R). The claim is the conclusion of an argument and the main point toward which all other elements of an argument converge. It seems like a topic sentence in a passage. Other elements support it and are subordinate to it. For example, it may be a proposal of a policy document. The ground is the element that directly supports the claim and it can be the fact, the oral testimony and the matter of common knowledge. The warrant is the connection between the claim and the ground. Since the warrant can be self-validating, we also need the backing which is the overarching principle and value. The warrant and the backing may be presuppositions underlying the communication. The qualifier is to express the force of the claim, which is represented by an adverb such as definitely, certainly and probably. The rebuttal represents exceptional circumstance that may undermine the force of the arguments. The B-W-G-C-Q-R argument schema provides a systematic and complete discourse analysis and reveals a combination of the semantic, pragmatic and textual meaning of a text.

Therefore, Malcolm Williams thinks the process of the translation quality assessment is radically to determine whether the basic arguments $(\mathrm{B}, \mathrm{W}, \mathrm{G}, \mathrm{C}, \mathrm{Q}, \mathrm{R})$ in the source text (ST) are accurately rendered in the target text (TT). That is to say, when we assess the translation quality, firstly we need to describe the respective argument schema of ST and TT and then make a comparison of two argument schemas. Finally, we can draw the evaluation of the translation quality. Nevertheless, this is not enough to give a scientific assessment because some other factors should be also taken into consideration.

\section{Argumentation parameters and weighted ARTRAQ grid}

To improve the model, Malcolm Williams selects other parameters based on the stylistics and designs the weighted ARTRAQ grid (Table 1).

Firstly, in addition to the argument schema belonging to the method of macro-analysis, Malcolm Williams thinks some parameters of micro-analysis should also be included in the model. According to this, conjunctives/other inference indicators become ideal parameters because they can signpost the progression of ideas in a text and demonstrate the logical connections of different arguments. Another reason is that these words are always easily misinterpreted even by experienced translators, which have a direct influence on the cohesion in a text. 
Secondly, to establish relations between the connectors and the argument structure of a high level, the author develops the propositional functions as another parameter. Propositional functions give a clearer relationship between different propositions, which can be clarified into six types: generalization, clarification, elaboration, consequence, qualification and explanation. Whether the translators can show these propositional functions in their TT determines their translation quality.

Table 1 Weighted ARTRAQ Grid (Example)

\begin{tabular}{|c|c|c|c|c|}
\hline Parameter & Weight(/10) & $\begin{array}{c}\text { Minimum } \\
\text { requirement }\end{array}$ & $\begin{array}{c}\text { Quality } \\
(/ 10)\end{array}$ & Score $(/ 100)$ \\
\hline Argument schema & 3 & $(10) 30$ & 9 & 27 \\
\hline $\begin{array}{c}\text { Propositional } \\
\text { functions/conjunctives/ } \\
\text { other inference } \\
\text { indicators }\end{array}$ & 2 & $(8) 16$ & 7 & 14 \\
\hline Narrative strategy & 2 & $(8) 16$ & 7 & 14 \\
\hline typography & 1 & $(8) 8$ & 6 & 6 \\
\hline Terminology & 2 & $(8) 16$ & 6 & 12 \\
\hline Total & 10 & 86 & - & 73 \\
\hline Grade & \multicolumn{3}{|c|}{ Substandard } \\
\hline
\end{tabular}

The third parameter is the narrative strategy, which has two main forms: depersonalization and qualifiers. The former one means that the narrator should hide his or her "presence" in the scientific texts to show the objectivity of the science. The latter one means the narrator may use some qualifiers such as "I would like to suggest" or "it seems" to show the caution of the science. When we translate these elements, we must render it accurately.

In Malcolm Williams' theory, argument schema, propositional functions/conjunctives/other inference indicators and the narrative strategy mentioned above are named core parameters, which can mainly reflect the translation quality. To fit for different types of text, the author also creates some use-specific parameters such as typography and terminology, the most common parameters.

Remarkably, on the basis of Larose framework, Malcolm Williams also applies the mathematical method to make the assessment result straightforward. He gives various weights to different parameters from 1 to 10 according their importance. Since the argument schema is critical, its weigh can reach 3 or 4 . The quality of every parameter is rated from 0 to $10(10=$ excellent $8=$ very satisfactory $6=$ satisfactory $4=$ fair $2=$ poor). After multiplying the weight by the quality, we can get the score of every parameter. And the total score is the summation of all the single score, which represents the final result. By comparing the final score with the minimum requirement (acceptable score of the translation quality), we can determine which standard the translator belong to, maximum standard, information standard, minimum standard or substandard.

\section{A revised weighted ARTRAQ grid for assessing the translation quality in the translation contest}

By applying the argumentation theory and the quantitative method, this model can provide a scientific and intuitive translation quality assessment, which is appropriate for the translation contests. But combined with the practical requirement of the judgment, there is a need to optimize the parameters when the model is applied into the translation quality assessment in the translation contest. Due to the limited judging time, it is essential to reduce the number of the parameters to ensure the efficiency. Firstly, we can cut the typography because everyone has the similar performance in this point in the translation contest. And then we may change the narrative strategy into figure of speech, considering that the latter one is a more important point in the contest. Furthermore, we choose to omit the 
minimum requirement and grade for the reason that the contest only needs a ranking list by the score. Finally we can get a revised weighted ARTRAQ grid for assessing the translation quality in the translation contest.

Generally, the process of the model can be summarized as follows:

(1)According to the text, set a proper weight for every parameter.

(2)Analyze the ST and TT based on argumentation theory to find the B-W-G-C-Q-R and describe the respective argument schema. Check whether the argument schema in the ST is rendered accurately into the TT and give a point.

(3)Identify the type of the propositional function of the ST. Check whether it is shown in the TT and give a point.

(4)Locate all the conjunctives/other inference indicators in the ST. Check whether it is translated in the TT and give a point.

(5)Analyze the figure of speech used in the ST. Check whether it has the corresponding expressions in the TT and give a point.

(6)Locate all the terminology in the ST. Check whether it is translated accurately in the TT and give a point.

(7) Calculate the total scores.

The following is a case of the application of the revised model:

Table 2 revised weighted ARTRAQ grid for assessing the translation quality in the translation contest

\begin{tabular}{|c|c|c|c|}
\hline Parameter & Weight(/10) & Quality (/10) & Score(/100) \\
\hline Argument schema & 3 & 8 & 24 \\
\hline $\begin{array}{c}\text { Propositional } \\
\text { functions/conjunctives } \\
\text { /other inference } \\
\text { indicators }\end{array}$ & 3 & 6 & 18 \\
\hline Figure of speech & 2 & 6 & 12 \\
\hline Terminology & 2 & 8 & 16 \\
\hline Total & 10 & - & 70 \\
\hline
\end{tabular}

If the point of every parameter of a translation is given as Table 2, then the score of the translation quality will be graded as 70 .

\section{Conclusion}

According to the argumentation theory, the argumentation in the text can be regarded as a main presentation form of knowledge and meaning. In view of this, Malcolm Williams thinks that the translation quality assessment is essentially the comparison of the argument structure between ST and TT. And he proposes a concept of the argument schema to describe the argument structure of the texts and develops Malcolm Williams' argumentation-centered translation quality assessment model. In this model, he also adopts other factors such as conjunctives, propositional functions and the narrative strategy to perfect the model. With both macro-analysis and micro-analysis, the model has a very systematic and scientific advantage. Besides, to show a straghtforward result of the asseessment, he introduces the quantitative approach and build the weighted ARTRAQ grid, superior to other translation quality assessment model. Therefore, Malcolm Williams' argumentation-centered translation quality assessment model can provide a solid theoretical basis for the translation quality assessment in the translation contest and we can build a revised weighted ARTRAQ grid to assess the translation quality in the translation contest according to the practical requirement of the judgment. 
When we apply the model to evaluate the translation quality in the translation contest, we should notice its conditions of application and optimizing the parameters according to differents types of texts.

However, there are still some defects further improved in the future. For example, the model can't be regarded as an absolutely objective method since it's a bit subjective in the process of endowing the weight to different parameters. Therefore it's necessary to continue to amend its parameters from the perspective of the practicability in the translation contests and do more experiments to find the proper weights for different parameters.

\section{Acknowledgements}

This research is sponsored by Jinan University's Scientific Research Creativeness Cultivation Project for Outstanding Undergraduates Recommended for Post-graduate Study.

\section{References}

[1] Al-Qinai J. Translation Quality Assessment. Strategies, Parametres and Procedures[J]. Meta, 2000, 45(3):497-519.

[2] Guangjun $\mathrm{Wu}$. An introduction to the translation quality assessment: an argumentation-centred approach [J].Foreign languages research, 2006(4):72-74.

[3] House J. Translation Quality Assessment: Past and Present[M]. Translation: A Multidisciplinary Approach. Palgrave Macmillan UK, 2014.

[4] Shouyi Fan. Fuzzy Mathematics and Translation Quality Evaluation[J]. Chinese Translators Journal, 1987(04).

[5] Toulmin S, Rieke R, Janik A. An Introduction to Reasoning[M]. A new introduction to Greek I. :562-4.

[6] Williams M. An argumentation-centred approach to translation quality assessment[J]. University of Ottawa, 2001. 